\title{
Corticopallidal Connectome of the Globus Pallidus Externus in Humans: An Exploratory Study of Structural Connectivity Using Probabilistic Diffusion Tractography
}

\author{
(D)S.S. Grewal, (DV.M. Holanda, and (D)E.H. Middlebrooks
}

\begin{abstract}
BACKGROUND AND PURPOSE: Electrophysiologic abnormalities of the globus pallidus externus have been shown in several disease processes including Parkinson disease, dystonia, and Huntington disease. However, the connectivity, nuclear structure, and function of the globus pallidus externus are still not well-understood. Increasing evidence for the existence of direct corticopallidal connections challenges traditional understanding of the connectivity of the globus pallidus externus; nevertheless, these corticopallidal connections have yet to be fully characterized in humans. The objective of this study was to assess the corticopallidal connections of the globus pallidus externus by means of probabilistic diffusion-weighted MR imaging tractography using high-resolution, multishell data.
\end{abstract}

MATERIALS AND METHODS: Imaging data from the open-access Human Connectome Project data base were used to perform probabilistic tractography between the globus pallidus externus and the cerebral cortex using 34 distinct cortical regions. Group averages were calculated for normalized percentages of tracts reaching each of the cortical targets, and side-to-side comparison was made.

RESULTS: Cortical connectivity was demonstrated between the globus pallidus externus and multiple cortical regions, including direct connection to putative sensorimotor, associative, and limbic areas. Connectivity patterns were not significantly different between the right and left hemispheres with the exception of the frontal pole, which showed a greater number of connections on the right $(P=.004)$.

CONCLUSIONS: Our in vivo study of the human globus pallidus externus using probabilistic tractography supports the existence of extensive corticopallidal connections and a tripartite functional division, as found in animal studies. A better understanding of the connectivity of the globus pallidus externus may help to understand its function and elucidate the effects of programming the higher contacts in pallidal deep brain stimulation.

ABBREVIATIONS: $\mathrm{DBS}=$ deep brain stimulation; $\mathrm{GPe}=$ globus pallidus externus

$\mathrm{T}$ he globus pallidus externus (GPe) is the lateral component of the globus pallidus known to have extensive connections with other basal ganglia nuclei. ${ }^{1}$ Traditionally thought to primarily function in motor control, ${ }^{2}$ more recent evidence has challenged this belief, showing additional function in associative and limbic processes. $^{3-5}$ GPe dysfunction has been shown in several disease processes, including a decreased spontaneous discharge rate in

Received February 11, 2018; accepted after revision August 7.

From the Departments of Neurosurgery (S.S.G., E.H.M.) and Radiology (E.H.M.), Mayo Clinic, Jacksonville, Florida; and Center of Neurology and Neurosurgery Associates (V.M.H.), BP-A Beneficência Portuguesa de São Paulo, São Paulo, Brazil.

Data were provided, in part, by the Human Connectome Project, WU-Minn Consortium (Principal Investigators: David Van Essen and Kamil Ugurbil, 1U54MH091657) funded by the 16 National Institutes of Health institutes and centers that support the National Institutes of Health Blueprint for Neuroscience Research, and by the McDonnell Center for Systems Neuroscience at Washington University.

Please address correspondence to Erik H. Middlebrooks, MD, Mayo Clinic, Department of Neurosurgery, 4500 San Pablo Rd, Jacksonville, FL 32224;

e-mail: Middlebrooks.Erik@mayo.edu

http://dx.doi.org/10.3174/ajnr.A5816
Parkinson disease ${ }^{6}$ and dystonia ${ }^{7}$ and an increased spontaneous discharge rate in Huntington disease. ${ }^{8}$ These findings suggest an important role of the GPe in the pathophysiology of several disease processes and a potential clinical relevance. ${ }^{9,10}$

In 2003, Silberstein et $\mathrm{al}^{11}$ showed that the local field potentials recorded in the globus pallidus externus in patients who underwent deep brain stimulation (DBS) were greater in patients with Parkinson disease $(11-30 \mathrm{~Hz})$ than in those with dystonia (4-10 $\mathrm{Hz}$ ). This finding indicated the importance of understanding the GPe connections to help in elucidating the mechanistic basis of DBS when treating these diseases. Fifteen years later, the connectivity, nuclear structure, and function of the GPe are still not well-understood. ${ }^{1,5}$

Traditionally, the basal ganglia circuitry has been thought to involve 2 pathways, a "direct" and an "indirect" pathway, with the striatum being the main input source for cortical efferents. ${ }^{12,13}$ Nevertheless, this model was challenged with the postulation of a "hyperdirect" pathway, connecting the subthalamic nucleus to 
the cortex. ${ }^{14-16}$ Subsequently, there was increasing work in both animal and in vivo human models highlighting the existence of direct corticopallidal connections; however, these corticopallidal connections specific to the globus pallidus externus have yet to be fully characterized in humans. ${ }^{17-23}$ The objective of this study was to assess corticopallidal connections of the GPe by means of probabilistic diffusion-weighted MR imaging tractography, using high-resolution, multishell data.

\section{MATERIALS AND METHODS Data Acquisition}

Imaging data from the open-access Human Connectome Project data base were used (https://www.humanconnectome.org). Twenty healthy subjects were consecutively selected from the data base. Full imaging acquisition details are freely available at the Human Connectome Project data base Web site (https:// www.humanconnectome.org), but briefly, the imaging was obtained using a modified 3T Magnetom Skyra scanner (Siemens, Erlanger, Germany). The multidirection diffusion-weighted imaging used a spin-echo EPI sequence with an isotropic resolution of $1.25 \mathrm{~mm}^{3}$. Diffusion b-values were acquired at 1000, 2000, and $3000 \mathrm{~s} / \mathrm{mm}^{2}$ in 90 diffusion directions for each shell. A multiband factor of 3 with 6/8 phase partial Fourier was used, and 111 axial slices were obtained with no gap. The parameters were the following: $\mathrm{TR}=5520 \mathrm{~ms}, \mathrm{TE}=89.5 \mathrm{~ms}$, flip angle $=78^{\circ}$. Images were obtained in both the left-to-right and right-to-left phase-encoding directions. Total imaging time for the diffusion data was 59 minutes. The T1-weighted 3D MPRAGE structural scan used an isotropic resolution of $0.7 \mathrm{~mm}^{3}$. Additional acquisition parameters included the following: $\mathrm{TR}=2400 \mathrm{~ms}$, $\mathrm{TE}=2.14 \mathrm{~ms}$, $\mathrm{TI}=$ $1000 \mathrm{~ms}$, flip angle $=8^{\circ}$, generalized autocalibrating partially parallel acquisition $=2$, and bandwidth $=210 \mathrm{~Hz} / \mathrm{Px}$ for an acquisition time of 7 minutes and 40 seconds.

Preprocessing of the diffusion data included realignment, $B_{0}$ image-intensity normalization, EPI distortion correction with the FSL Topup algorithm (https://fsl.fmrib.ox.ac.uk/fsl/fslwiki/ topup), gradient nonlinearity correction, and eddy current correction. ${ }^{24}$ The diffusion data were co-registered to the structural MPRAGE images using a boundary-based registration method (BBR) implemented in the FMRIB Linear Registration Tool (FLIRT; http://www.fmrib.ox.ac.uk/fslwiki/FLIRT) followed by the "bbregister" function in FreeSurfer (https://surfer.nmr.mgh. harvard.edu). All preprocessing steps used standard options as detailed in the Human Connectome Project data base preprocessing pipeline. ${ }^{24}$

\section{Data Processing}

Segmentation of the MPRAGE data was performed with FreeSurfer (https://surfer.nmr.mgh.harvard.edu). The left and right cerebral cortices were segmented into 34 distinct volumes of interest in FreeSurfer based on the Desikan-Killiany cortical atlas using the FreeSurfer mri_label2vol function. ${ }^{25}$ VOIs were also generated of the right and left GPe in the Montreal Neurological Institute template space from the Montreal Neurological Institute Basal Ganglia Human Area Template. ${ }^{26}$ The GPe VOIs were then transformed from Montreal Neurological Institute space to Free-
Surfer space using a linear registration implemented in FSL FLIRT.

Voxelwise diffusion parameters were estimated via a Markov Chain Monte Carlo sampling implemented in FSL bedpost (http://fsl.fmrib.ox.ac.uk/fsl/fslwiki/FDT/UserGuide\#BEDPOSTX). A multifiber approach (2 fiber orientations) was used along with a multishell model. Next, probabilistic tractography was performed using the FSL probtrackx2 function (https://fsl.fmrib.ox.ac.uk/ fsl/fslwiki/FDT/UserGuide) with the network option to estimate the connectivity between each GPe VOI and the 34 ipsilateral cortical targets. Standard tracking parameters include a 0.2 curvature threshold, 2000 steps per sample, a step length of $0.5 \mathrm{~mm}$, and 5000 samples per side.

\section{Data Analysis}

For each subject, the weighted connectivity was calculated between each of the 34 cortical ROIs and the ipsilateral GPe ROI by means of bidirectional fiber tracking between the GPe ROIs and each cortical target ROI. Because the distance traveled has a positive correlation with fiber-tract uncertainty, the tracts were normalized by the distance traveled, as implemented in FSL probtrackx2. To account for potential tracking bias due to variable sizes of the ROIs, we normalized the connectivity matrix by the surface area of the ROIs according to the method proposed by Hagmann et al. ${ }^{27}$ The number of paths was normalized among all patients by dividing the total number of paths by the waytotal for each subject to facilitate between-subject comparison. Group averages were calculated for normalized percentages of tracts reaching each of the cortical targets. In an attempt to account for false fibers generated by probabilistic tractography, a normalized fiber threshold level was set to exclude all target VOIs. Appropriate threshold levels remain a point of contention in the literature; therefore, we have selected a probabilistic threshold that is in the range of that used in prior studies of $\geq 2 \%$ of the total $(98 \%$ confidence level) in either hemisphere. ${ }^{17,28}$ The VOIs exceeding the threshold were used to generate a connectogram for both the left and right GPe in Matlab 9.1 (MathWorks, Natick, Massachusetts). Last, differences between the connectivity profile of the left and right GPe were assessed for each cortical target in GraphPad Prism 7.0 software (GraphPad Software, San Diego, California) by means of a Mann-Whitney $U$ test to account for non-normality in distribution.

\section{RESULTS}

Of the 34 cortical targets evaluated (Table), 17 exceeded the fiber count threshold on either side (Figure). The right GPe exhibited maximal cortical connectivity to the frontal pole $(14.4 \% \pm 9.4 \%)$, and the left GPe had maximal cortical connectivity to the temporal pole $(10.5 \% \pm 8.0 \%)$. The greatest difference in the connectivity profile between sides was related to the frontal pole $(14.4 \% \pm 9.4 \%$ on the right and $7.7 \% \pm 5.4 \%$ on the left $)$, insula $(11.0 \% \pm 4.2 \%$ on the right and $8.5 \% \pm 4.6 \%$ on the left $)$, and entorhinal cortex $(5.1 \% \pm 4.4 \%$ on the right and $7.4 \% \pm 6.6 \%$ on the left). The side-to-side variation was also only significant for the frontal pole $(P=.004)$. The remaining targets showed no significant variation between sides $(P>.05)$. 
Cortical ROIs meeting the tract threshold for connectivity to the globus pallidus externus for both hemispheres ${ }^{a}$

\begin{tabular}{lccc}
\hline \multicolumn{1}{c}{ Cortical Region } & $\begin{array}{c}\text { Right Hemisphere } \\
\text { (\% Tract Probability) (SD) }\end{array}$ & $\begin{array}{c}\text { Left Hemisphere } \\
\text { (\% Tract Probability) (SD) }\end{array}$ & P Value \\
\hline Frontal pole & $14.4 \%(9.4 \%)$ & $7.7 \%(5.4 \%)$ & .004 \\
Insula & $11.0 \%(4.2 \%)$ & $8.5 \%(4.6 \%)$ & .1 \\
Temporal pole & $9.2 \%(7.7 \%)$ & $10.5 \%(8.0 \%)$ & .49 \\
Medial orbitofrontal & $6.7 \%(5.9 \%)$ & $8.3 \%(4.9 \%)$ & .21 \\
Entorhinal & $5.1 \%(4.4 \%)$ & $7.4 \%(6.6 \%)$ & .22 \\
Superior frontal & $4.3 \%(3.5 \%)$ & $4.8 \%(3.2 \%)$ & .56 \\
Pars orbitalis & $3.8 \%(2.6 \%)$ & $3.2 \%(2.6 \%)$ & .4 \\
Lateral orbitofrontal & $3.8 \%(3.9 \%)$ & $4.1 \%(3.9 \%)$ & .72 \\
Paracentral & $3.4 \%(2.9 \%)$ & $3.9 \%(2.9 \%)$ & .5 \\
Postcentral & $3.2 \%(2.2 \%)$ & $4.3 \%(2.2 \%)$ & .1 \\
Pars triangularis & $3.1 \%(4.1 \%)$ & $3.2 \%(3.7 \%)$ & .96 \\
Precentral & $2.9 \%(1.9 \%)$ & $4.4 \%(2.5 \%)$ & .07 \\
Rostral anterior cingulate & $2.7 \%(2.8 \%)$ & $1.2 \%(1.2 \%)$ & .06 \\
Superior parietal & $2.3 \%(1.3 \%)$ & $2.9 \%(1.2 \%)$ & .1 \\
Rostral middle frontal & $2.3 \%(1.4 \%)$ & $2.0 \%(1.2 \%)$ & .83 \\
Isthmus cingulate & $2.2 \%(1.2 \%)$ & $3.5 \%(3.2 \%)$ & .27 \\
Parahippocampal & $1.4 \%(1.2 \%)$ & $2.1 \%(1.5 \%)$ & .12 \\
\hline
\end{tabular}

${ }^{a}$ The $P$ value is reported for left-to-right tract comparison.

\section{DISCUSSION}

Our probabilistic tractography study highlights the potential widespread direct cortical connectivity of the GPe. As predicted from prior animal functional studies, direct cortical connections were found between the GPe and areas functioning in sensorimotor, associative, and limbic tasks. ${ }^{3,4}$ These direct corticopallidal connections challenge the traditional hypotheses regarding the GPe as merely a subcortical relay within the indirect pathway of the basal ganglia.

Initial descriptions of basal ganglia connectivity were largely based on the work of DeLong ${ }^{13}$ and Albin et $\mathrm{al}^{12}$, elucidated through a combination of both immunohistochemistry and in situ hybridization techniques. ${ }^{12,13}$ These models defined 2 corticofugal networks, a direct and indirect pathway of basal ganglia circuitry, and considered the dorsal striatum as the main input area for cortical efferent neurons to these pathways. ${ }^{12}$ More recently, a third network, a hyperdirect pathway, has been discovered in which glutamatergic cortical efferents synapse directly on the subthalamic nucleus. ${ }^{16}$ The existence of this hyperdirect pathway challenged the traditional hypotheses of basal ganglia connectivity. Indeed, evidence has mounted for additional subpopulations of efferent neurons with direct cortical connection to basal ganglia nuclei outside the dorsal striatum, including the GPe. ${ }^{17-23}$

Direct cortical connection to the GPe was described as early as 1977 in primates. ${ }^{29}$ It was again described in an anterograde tracing study in rodents, linking the precentral cortex of rodents to the GPe ${ }^{20}$ Additional studies have also described both cholinergic and GABAergic neurons within the GPe, sending direct efferent connections to the cortex. ${ }^{18,21}$ One in vivo human study used constrained spherical deconvolution to assess the existence of a corticopallidal network with connections from the GP to Brodmann areas 2, 11, 46, 48, 6, 4, and 5; however, distribution of fiber densities between areas was not assessed. ${ }^{19}$ Using a similar technique, Cacciola et al $^{17}$ described 2 separate corticopallidal networks: an anatomic pallidal-temporal network involving the hippocampus and amygdala and a sensorimotor-pallidal network mainly involving the precentral, postcentral, and paracentral gyri, in addition to higher order functional areas, such as the superior frontal gyrus. Unfortunately, due to grouping the GPe and globus pallidus internus as 1 ROI (pallidum), it is uncertain how the GPe and globus pallidus internus connectivity profiles differ. ${ }^{17}$ Our current study clarifies the distribution and density of corticopallidal connections specifically to the GPe.

The GPe is classically considered as part of the indirect pathway and extensively connected to deep brain structures. ${ }^{1,13}$ Animal studies of bicuculline injections into various regions of the GPe have elicited behavioral disturbances that can be functionally segmented into 3 zones: the ventrolateral "sensorimotor territory," the middle "associative territory," and the anterior ventral "limbic territory." 3,4 To assess the potential role of direct corticopallidal connections in these functional areas, prior studies used rabies virus injections to trace these potential connections. ${ }^{3,4}$ An injection into the limbic GPe labeled areas with known cortical projections: the rostromedial prefrontal cortex, insula, and orbitofrontal cortex, among others. ${ }^{3,4}$ Injection in the associative GPe labeled areas with known cortical projections to the supplementary motor area and pre-supplementary motor area as well as the associative parietal, motor, and premotor cortices. Finally, injection into the sensorimotor GPe labeled areas with known cortical projections to the premotor, primary motor, and somatosensory cortices. $^{3,4}$

In line with these animal studies, we were able to illustrate GPe direct cortical connectivity to similar putative associative, sensorimotor, and limbic areas. ${ }^{3,4}$ Direct cortical connections corresponding to the limbic GPe include the rostral middle frontal region (presumed dorsolateral prefrontal cortex), entorhinal cortex, parahippocampal gyrus, isthmus of the cingulate cortex, insula, and orbitofrontal cortex. ${ }^{3,4}$ Likewise, associative connections to the supplementary motor area/pre-supplementary motor area regions, superior parietal cortex, primary motor cortex, and premotor cortex were found. Last, as suggested by the sensorimotor function, we found corticopallidal connections between the GPe and the premotor, primary motor, and somatosensory cortices.

Animal studies have revealed attention deficits and hyperactivity induced by regional damage to the GPe, raising the possibility of a GPe role in the symptoms of attention deficit/hyperactivity disorder. ${ }^{3,4}$ The symptomatology of attention deficit/ hyperactivity disorder would suggest dysfunction in attention processing as well as motor planning. Although the role of the frontal pole is not well-understood, it has been implicated in the process of managing multiple cognitive tasks and attention. ${ }^{30}$ Spatial attention has also been attributed to function within the premotor and dorsolateral prefrontal cortices. ${ }^{31,32}$ Additionally, the rostral cingulate and supplementary motor area, both showing GPe connectivity in our study, have been implicated in the hyperactive symptoms seen in attention deficit/hyperactivity dis- 


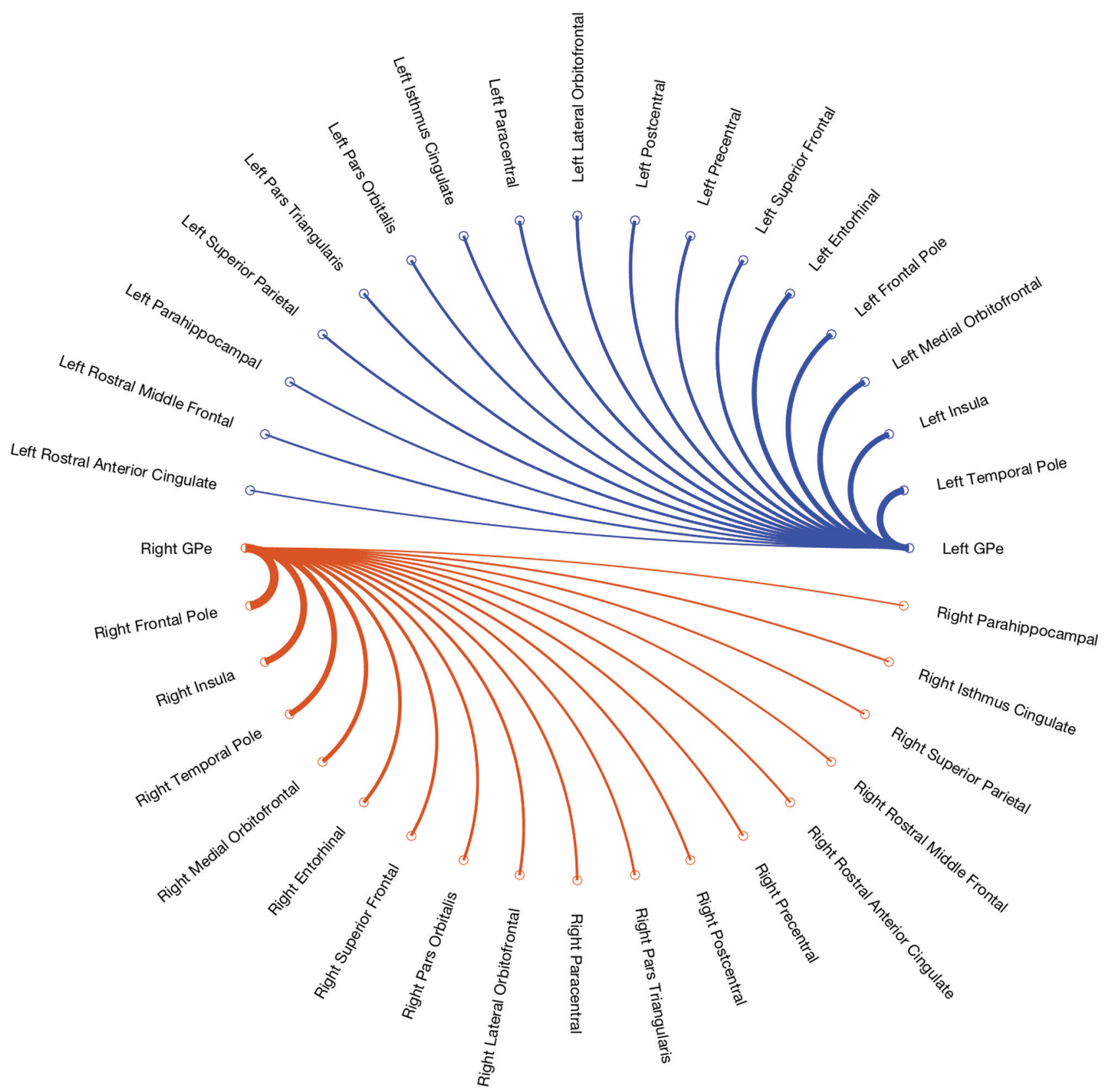

FIGURE. Connectogram showing the connectivity profile for the right and left globus pallidus externus. Lines are weighted by tract count.

order. $^{33}$ Taken together, the connectivity between the GPe and these cortical regions in our study would corroborate the attention and hyperactivity symptoms elicited in animal studies of the GPe. $^{3,4}$

Animal studies have also implicated the GPe in movement symptoms, such as dyskinesia, as well as stereotyped behavioral symptoms. Although they are not fully understood, it has been suggested that the ventral pallidum and striatal projections to the orbitofrontal cortex, insula, anterior cingulate, and amygdalohippocampal complex (regions functioning in the processing of motivation and emotion) may facilitate such behavioral symptoms. ${ }^{3,34-40}$ In addition to these known projections from other portions of the striatum, our study suggests the existence of direct corticopallidal connections originating from the GPe to similar regions, which could also contribute to the behaviors observed in obsessive-compulsive disorder and Tourette syndrome. Support that these symptoms are also mediated through the GPe includes reports that pallidal lesions can result in obsessive-compulsivetype behavior. ${ }^{41,42}$ Additionally, DBS of the GPe has been shown to reduce tics in patients with Tourette syndrome. ${ }^{43,44}$

Broad connectivity of the GPe to primary and secondary sensorimotor regions, including the superior frontal, precentral, and postcentral gyri may play an additional role in mediating symptoms of movement disorders, such as Parkinson disease, Huntington disease, and dystonia. ${ }^{45-47}$ In fact, DBS of the GPe has been shown as a potential treatment for both Parkinson disease and dystonia. ${ }^{46,47}$ Whether these treatment effects are, in part, mediated by direct corticopallidal connectivity remains to be determined, but they could potentially provide an additional treatment targeting for these debilitating conditions. 
In summary, the connectivity and function of the GPe are not as well-understood compared with other components of the basal ganglia. ${ }^{1}$ Current evidence supports a role of the GPe in limbic, associative, and sensorimotor processes. ${ }^{3,4}$ Animal tracer studies have demonstrated connectivity of the GPe to additional sites in the basal ganglia that have secondary cortical connections to areas of matching function ${ }^{3,4}$; however, we were also able to demonstrate primary connections to similar cortical areas from corticopallidal fibers. A more complete understanding of the function of the GPe may help shed light on its role in pathologic conditions, including Parkinson disease, Huntington disease, obsessive-compulsive disorder, attention deficit/hyperactivity disorder, and dystonia. ${ }^{6,8,9}$ In the future, the knowledge of connectivity beyond the motor network may also reveal a role of the GPe in other disorders of the limbic and associative networks.

Several limitations of this study are noteworthy. Firstly, limitations of diffusion-based tractography itself include the inability to determine the directionality (afferent-efferent) of fiber tracts, the limited resolution of crossing fibers, and the potential for "false fibers." ${ }^{48,49}$ Probabilistic tractography is a more sensitive and robust method compared with deterministic tractography; however, probabilistic tractography is associated with a higher incidence of false fiber detection. ${ }^{50}$ Additionally, the selection of thresholds for fiber tract probabilities has pitfalls. ${ }^{51}$ For instance, a very small bundle of true fibers may be rejected as a statistical error simply due to the small number of fibers, effectively biasing the results to larger fiber bundles. Second, the diffusion-based tractography method is unable to directly provide any functional information regarding the fiber tracts; therefore, the function of the identified tracts is merely speculative. Nevertheless, the anatomic connectivity illustrated in our study is well-aligned with functional deficits identified in prior animal studies.,

\section{CONCLUSIONS}

Our in vivo study of the human GPe using probabilistic tractography supports the existence of extensive corticopallidal connections. Direct cortical connections to putative sensorimotor, associative, and limbic areas support this tripartite functional division found in animal studies. A better understanding of the connectivity of the GPe may help to understand its function and elucidate the effects of programming the higher contacts in pallidal DBS. Further electrophysiologic studies are needed to investigate the significance of these connections and their ultimate role in basal ganglia circuitry.

\section{REFERENCES}

1. Hegeman DJ, Hong ES, Hernández VM, et al. The external globus pallidus: progress and perspectives. Eur J Neurosci 2016;43:1239-65 CrossRef Medline

2. Mouton S, Xie-Brustolin J, Mertens P, et al. Chorea induced by globus pallidus externus stimulation in a dystonic patient. Mov Disord 2006;21:1771-73 CrossRef Medline

3. François C, Grabli D, McCairn K, et al. Behavioural disorders induced by external globus pallidus dysfunction in primates, II: anatomical study. Brain 2004;127:2055-70 CrossRef Medline

4. Grabli D, McCairn K, Hirsch EC, et al. Behavioural disorders induced by external globus pallidus dysfunction in primates, I: behavioural study. Brain 2004;127:2039-54 CrossRef Medline

5. Gittis AH, Berke JD, Bevan MD, et al. New roles for the external globus pallidus in basal ganglia circuits and behavior. $J$ Neurosci 2014;34:15178-83 CrossRef Medline

6. Filion M, Tremblay L. Abnormal spontaneous activity of globus pallidus neurons in monkeys with MPTP-induced parkinsonism. Brain Res 1991;547:142-51 Medline

7. Stoessl AJ, Lehericy S, Strafella AP. Imaging insights into basal ganglia function, Parkinson's disease, and dystonia. Lancet 2014;384: 532-44 CrossRef Medline

8. Starr PA, Kang GA, Heath S, et al. Pallidal neuronal discharge in Huntington's disease: support for selective loss of striatal cells originating the indirect pathway. Exp Neurol 2008;211:227-33 CrossRef Medline

9. Starr PA, Rau GM, Davis V, et al. Spontaneous pallidal neuronal activity in human dystonia: comparison with Parkinson's disease and normal macaque. J Neurophysiol 2005;93:3165-76 CrossRef Medline

10. Nambu A, Chiken S, Shashidharan P, et al. Reduced pallidal output causes dystonia. Front Syst Neurosci 2011;5:89 CrossRef Medline

11. Silberstein P, Kühn AA, Kupsch A, et al. Patterning of globus pallidus local field potentials differs between Parkinson's disease and dystonia. Brain 2003;126:2597-608 CrossRef Medline

12. Albin RL, Young AB, Penney JB. The functional anatomy of basal ganglia disorders. Trends Neurosci 1989;12:366-75 CrossRef Medline

13. DeLong MR. Primate models of movement disorders of basal ganglia origin. Trends Neurosci 1990;13:281-85 CrossRef Medline

14. Brunenberg EJ, Moeskops $\mathrm{P}$, Backes WH, et al. Structural and resting state functional connectivity of the subthalamic nucleus: identification of motor STN parts and the hyperdirect pathway. PLoS One 2012; 7:e39061 CrossRef Medline

15. Forstmann BU, Keuken MC, Jahfari S, et al. Cortico-subthalamic white matter tract strength predicts interindividual efficacy in stopping a motor response. Neuroimage 2012;60:370-75 CrossRef Medline

16. Nambu A, Tokuno H, Takada M. Functional significance of the cortico-subthalamo-pallidal 'hyperdirect' pathway. Neurosci Res 2002; 43:111-17 CrossRef Medline

17. Cacciola A, Calamuneri A, Milardi D, et al. A connectomic analysis of the human basal ganglia network. Front Neuroanat 2017;11:85 CrossRef Medline

18. Chen MC, Ferrari L, Sacchet MD, et al. Identification of a direct GABAergic pallidocortical pathway in rodents. Eur J Neurosci 2015; 41:748-59 CrossRef Medline

19. Milardi D, Gaeta M, Marino S, et al. Basal ganglia network by constrained spherical deconvolution: a possible cortico-pallidal pathway? Mov Disord 2015;30:342-49 CrossRef Medline

20. Naito A, Kita H. The cortico-pallidal projection in the rat: an anterograde tracing study with biotinylated dextran amine. Brain Res 1994;653:251-57 CrossRef Medline

21. Saunders A, Oldenburg IA, Berezovskii VK, et al. A direct GABAergic output from the basal ganglia to frontal cortex. Nature 2015;521: 85-89 CrossRef Medline

22. Neumann WJ, Jha A, Bock A, et al. Cortico-pallidal oscillatory connectivity in patients with dystonia. Brain 2015;138:1894-906 CrossRef Medline

23. Hoover JE, Strick PL. Multiple output channels in the basal ganglia. Science 1993;259:819-21 CrossRef Medline

24. Glasser MF, Sotiropoulos SN, Wilson JA, et al; WU-Minn HCP Consortium. The minimal preprocessing pipelines for the Human Connectome Project. Neuroimage 2013;80:105-24 CrossRef Medline

25. Desikan RS, Ségonne F, Fischl B, et al. An automated labeling system for subdividing the human cerebral cortex on MRI scans into gyral based regions of interest. Neuroimage 2006;31:968-80 CrossRef Medline

26. Prodoehl J, Yu H, Little DM, et al. Region of interest template for the human basal ganglia: comparing EPI and standardized space approaches. Neuroimage 2008;39:956-65 CrossRef Medline

27. Hagmann $P$, Cammoun L, Gigandet X, et al. Mapping the structural 
core of human cerebral cortex. PLoS Biol 2008;6:e159 CrossRef Medline

28. van Wijk BC, Stam CJ, Daffertshofer A. Comparing brain networks of different size and connectivity density using graph theory. PLoS One 2010;5:e13701 CrossRef Medline

29. Leichnetz GR, Astruc J. The course of some prefrontal corticofugals to the pallidum, substantia innominata, and amygdaloid complex in monkeys. Exp Neurol 1977;54:104-09 CrossRef Medline

30. Koechlin E, Hyafil A. Anterior prefrontal function and the limits of human decision-making. Science 2007;318:594-98 CrossRef Medline

31. Corbetta M, Miezin FM, Dobmeyer S, et al. Selective and divided attention during visual discriminations of shape, color, and speed: functional anatomy by positron emission tomography. J Neurosci 1991;11:2383-402 CrossRef Medline

32. Boussaoud D. Attention versus intention in the primate premotor cortex. Neuroimage 2001;14:S40-45 CrossRef Medline

33. Takada M, Tokuno H, Hamada I, et al. Organization of inputs from cingulate motor areas to basal ganglia in macaque monkey. Eur J Neurosci 2001;14:1633-50 CrossRef Medline

34. Chikama M, McFarland NR, Amaral DG, et al. Insular cortical projections to functional regions of the striatum correlate with cortical cytoarchitectonic organization in the primate. J Neurosci 1997;17: 9686-705 CrossRef Medline

35. Haber SN, Kunishio K, Mizobuchi M, et al. The orbital and medial prefrontal circuit through the primate basal ganglia. J Neurosci 1995;15:4851-67 CrossRef Medline

36. Kunishio K, Haber SN. Primate cingulostriatal projection: limbic striatal versus sensorimotor striatal input. J Comp Neurol 1994;350: 337-56 CrossRef Medline

37. Ferry AT, Ongür D, An X, et al. Prefrontal cortical projections to the striatum in macaque monkeys: evidence for an organization related to prefrontal networks. J Comp Neurol 2000;425:447-70 CrossRef Medline

38. Friedman DP, Aggleton JP, Saunders RC. Comparison of hippocampal, amygdala, and perirhinal projections to the nucleus accumbens: combined anterograde and retrograde tracing study in the Macaque brain. J Comp Neurol 2002;450:345-65 CrossRef Medline

39. Russchen FT, Bakst I, Amaral DG, et al. The amygdalostriatal projections in the monkey: an anterograde tracing study. Brain Res 1985;329:241-57 CrossRef Medline

40. Fudge JL, Haber SN. Defining the caudal ventral striatum in primates: cellular and histochemical features. J Neurosci 2002;22: 10078-82 CrossRef Medline

41. Laplane D, Baulac M, Widlöcher D, et al. Pure psychic akinesia with bilateral lesions of basal ganglia. J Neurol Neurosurg Psychiatry 1984; 47:377-85 CrossRef Medline

42. Laplane D, Levasseur M, Pillon B, et al. Obsessive-compulsive and other behavioural changes with bilateral basal ganglia lesions: a neuropsychological, magnetic resonance imaging and positron tomography study. Brain 1989;112(Pt 3):699-725 CrossRef Medline

43. Filho OV, Ragazzo PC, Silva DJ, et al. Bilateral globus pallidus externus deep brain stimulation (GPe-DBS) for the treatment of Tourette syndrome: an ongoing prospective controlled study. Stereotact Funct Neurosurg 2007;85:42-43 CrossRef

44. Piedimonte F, Andreani JC, Piedimonte L, et al. Behavioral and motor improvement after deep brain stimulation of the globus pallidus externus in a case of Tourette's syndrome. Neuromodulation 2013;16:55-58; discussion 58 CrossRef Medline

45. Singh-Bains MK, Waldvogel HJ, Faull RL. The role of the human globus pallidus in Huntington's disease. Brain Pathol 2016;26: 741-51 CrossRef Medline

46. Vitek JL, Zhang J, Hashimoto T, et al. External pallidal stimulation improves parkinsonian motor signs and modulates neuronal activity throughout the basal ganglia thalamic network. Exp Neurol 2012; 233:581-86 CrossRef Medline

47. Houeto JL, Yelnik J, Bardinet E, et al; French Stimulation du Pallidum Interne dans la Dystonie Study Group. Acute deep-brain stimulation of the internal and external globus pallidus in primary dystonia: functional mapping of the pallidum. Arch Neurol 2007;64:1281-86 CrossRef Medline

48. Chung HW, Chou MC, Chen CY. Principles and limitations of computational algorithms in clinical diffusion tensor MR tractography. AJNR Am J Neuroradiol 2011;32:3-13 CrossRef Medline

49. Parker GD, Marshall D, Rosin PL, et al. A pitfall in the reconstruc tion of fibre ODFs using spherical deconvolution of diffusion MRI data. Neuroimage 2013;65:433-48 CrossRef Medline

50. Maier-Hein KH, Neher PF, Houde JC, et al. The challenge of mapping the human connectome based on diffusion tractography. Nat Commun 2017;8:1349 CrossRef Medline

51. Schlaier JR, Beer AL, Faltermeier R, et al. Probabilistic vs. deterministic fiber tracking and the influence of different seed regions to delineate cerebellar-thalamic fibers in deep brain stimulation. Eur J Neurosci 2017;45:1623-33 CrossRef Medline 